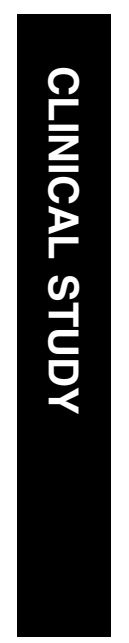

\title{
Use of a mydriatic cocktail with a wick for preoperative mydriasis in cataract surgery: a prospective randomised controlled trial
}

Department of

Ophthalmology, Jawaharlal Institute of Post graduate Medical Education and Research (JIPMER), Pondicherry, India

Correspondence: S Sengupta, Cornea and Refractory Surgery Services; Aravind Eye Hospital, Thavalakuppam, Pondicherry, India

Tel: 04132619 100; Fax: 04132618844 . Email: sengupta_sunny@ rediffmail.com

Received: 15 September 2008

Accepted in revised form: 12 January 2009

Published online: 6 March 2009

The authors declare no conflicting interests and no sources of support. This manuscript has not been submitted to any journal in the past and has never been published. It has not been presented in any meeting at present. However, it has been accepted as a free paper for the All India Ophthalmological Society conference to be held in February 2009

\begin{abstract}
Purpose To investigate the efficacy of a standardised wick soaked in a mydriatic cocktail regimen and compare it with the pre-existing regimen of topical dilating drops.

Design Prospective, two armed, randomised, double blind, parallel assignment efficacy study

Participants In all, 150 eyes of 150 patients were dilated using the dilating cocktail regimen by using a Weck cell sponge. The control group comprised of 75 eyes of 75 patients dilated using the conventional mydriatic regimen.

Interventions A specially prepared mydriatic cocktail regimen consisting of $1: 1: 1: 1$ ratio of $2.5 \%$ phenylephrine, $0.5 \%$ moxifloxacin, $1 \%$ cyclopentolate, and $0.03 \%$ flurbiprofen eye drops was prepared. A standard sized wick $(1 \mathrm{~cm} \times 2 \mathrm{~mm})$ made of Weck cell sponge was soaked in the cocktail for a minute and placed in the lower fornix with sterile forceps. In the control group, eyes were dilated with the above drops using topical instillation in every $15 \mathrm{~min}$. The mydriasis was measured at 15,30 , and $45 \mathrm{~min}$ after instillation using the Procyon pupillometer under scotopic conditions ( 0.04 lux). The intraoperative maintenance of mydriasis was measured with calipers after nucleus delivery and after intraocular lens implantation.

Main outcome measures Pupillary diameters at the end of 15, 30, and $45 \mathrm{~min}$, and intraoperative maintenance of mydriasis in the two groups were compared using unpaired $t$-test.
\end{abstract}

S Sengupta, K Subramoney, R Srinivasan, B Nongrum, V Agarwal, DG Pandian, T Suchismitha and S Kaliaperumal
Results There was a statistically significant difference $(P<0.001)$ in dilation achieved at the end of $45 \mathrm{~min}$ in both groups. There was also a significant difference of 1-mm dilation between the two groups after nucleus delivery $(P<0.001)$, and a difference of $1.41 \mathrm{~mm}$ after intraocular lens implantation, which was statistically significant $(P<0.001)$. Conclusions These results show that the mydriatic cocktail regimen delivered with a wick is a useful, economical, and timesaving regimen compared with the conventional method of preoperative mydriasis using topical drops.

Eye (2010) 24, 118-122; doi:10.1038/eye.2009.23; published online 6 March 2009

Keywords: mydriasis; cocktail; wick; cataract

Introduction

Pupillary dilation is an integral component of preoperative preparation for cataract surgery. This has traditionally been accomplished by topical instillation of mydriatic drops, such as $2 \%$ homatropine, $1 \%$ cyclopentolate, and $1 \%$ tropicamide eye drops with or without $2.5 \%$ phenylephrine eye drops. Other forms of drug delivery to effect pupillary dilation include ointment delivery, such as atropine eye ointment, intracameral mydriatic ${ }^{1}$ and mydrisert, and ${ }^{2}$ a sustained release formulation. A subconjunctival injection of mydricaine that consists of equal proportions of adrenaline, atropine, and lignocaine is also efficacious in dilation of patients with poor mydriasis. Topical 
non-steroidal anti-inflammatory drugs (NSAIDs), such as flurbiprofen or diclofenac, are also used along with the dilating regimen as they maintain the intraoperative mydriasis. $^{5-8}$ It is imperative to have adequate and sustained mydriasis and optimum corneal clarity during intraocular surgery. However, repeated instillation of mydriatic and anti-inflammatory ophthalmic solutions during preoperative dilation may affect compliance and may damage the corneal epithelium. Pupils are frequently dilated on the day before cataract surgery so as to enable fundus examination. This may, however, interfere with mydriasis on the day of surgery, termed pupillary fatigue. ${ }^{9}$

\section{Materials and methods}

In all, 225 eyes of 225 patients were included in this study, which was carried out over a period of 6 months. The study included all patients enrolled for cataract surgery with grade II and III nuclear sclerosis as per the Lens Opacification Classification System III (LOCS III). Patients expected to have poor mydriasis, such as diabetics, and those with pseudoexfoliation (PXF) were also included in the study. Patients with posterior synechiae due to long-standing uveitis, glaucoma, those with corneal pathologies, phacotoxic uveitis, and so on were excluded from this study. Individuals with systemic hypertension and ischaemic heart disease were also excluded owing to the use of phenylephrine in the cocktail regimen. All patients were dilated preoperatively during the outpatient visit using topical tropicamide and phenylephrine drops for evaluating the grade of cataract and examining the posterior segment. The degree of mydriasis was recorded. Among them, patients requiring cataract surgery were randomised into two groups.

\section{Randomisation}

Small pieces of folded card paper were placed in a bag with words 'trial' or 'control' written on them. Once the patient had consented to be a part of the study, a card was picked from the bag by a nurse who was not involved in the study. At the end of $45 \mathrm{~min}$, mydriasis was recorded on a photo slit lamp using a horizontal slit beam, by an independent observer who was blinded to the mydriatic regimen used. The operating surgeon was also blinded to the regimen used for mydriasis. It was not possible to conceal the arm of the trial from the patient because of the nature of the intervention.

\section{Intervention}

Group A contained 150 patients randomised to receive mydriatic cocktail delivered with a wick (Figure 1). A mydriatic cocktail was prepared in a sterile container using equal quantities of $2.5 \%$ phenylephrine, $1 \%$

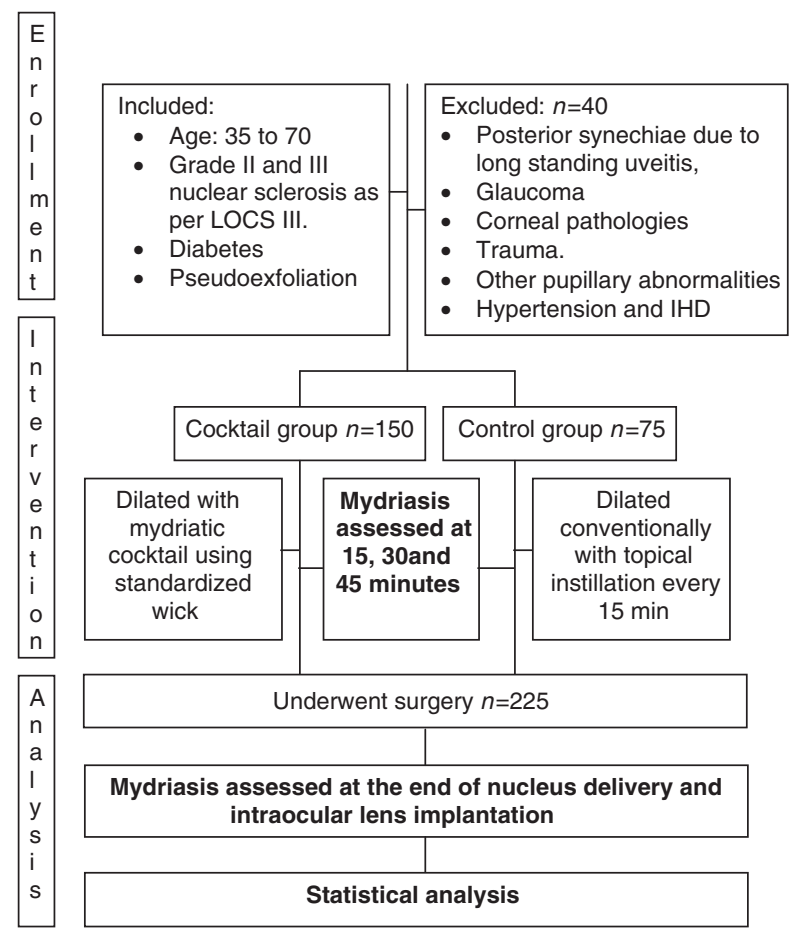

Figure 1 Flow plan showing enrolment, allocation, intervention, and analysis.

cyclopentolate, $0.5 \%$ moxifloxacin, and $0.03 \%$ flurbiprofen. All the solutions were prepared on the day of surgery using sterile precautions. Wicks were prepared from sterile Weck cell sponges that were cut using sterile scissors into $1 \mathrm{~cm} \times 2 \mathrm{~mm}$ rectangular strips and soaked in the cocktail solution for $1 \mathrm{~min}$. After using proparacaine to anaesthetise the ocular surface, the wicks were placed in the lower fornix using sterile forceps. The eyes were then taped to prevent the wicks from falling. In all, $4 \mathrm{ml}$ of cocktail solution was sufficient to dilate 10 patients simultaneously. Mydriasis was assessed with $0.5 \mathrm{~mm}$ intervals, using the procyon pupillometer under scotopic conditions (0.04 lux), at 15, 30, and $45 \mathrm{~min}$

Group B contained 75 patients randomised to receive the traditional regimen of dilating drops and served as controls for Group A. The drops used were 1\% cyclopentolate, $2.5 \%$ phenylephrine, $0.03 \%$ flurbiprofen, and $0.5 \%$ moxifloxacin. These were instilled topically as one drop from standard droppers. A single drop from each group was instilled every $3 \mathrm{~min}$ in succession, and the procedure was repeated every $15 \mathrm{~min}$ for $45 \mathrm{~min}$. The mydriasis was noted at 15, 30, and 45 min intervals.

On the basis of maximal horizontal pupillary diameter at $45 \mathrm{~min}$, patients were categorised into three groups:

Poor mydriasis: pupils $\leqslant 5 \mathrm{~mm}$ in size

Moderate mydriasis: pupils $>5$ but $<8 \mathrm{~mm}$ in size

Good mydriasis: pupils $\geqslant 8 \mathrm{~mm}$ in size 
All patients underwent phacoemulsification using a scleral tunnel incision by a single surgeon. The technique used was the divide and conquer technique in all cases. The intraoperative pupillary diameter was noted at two stages in the surgery, after nucleus extraction and intraocular lens (IOL) implantation. The pupillary size was measured using Castroviejo calipers. All patients had foldable hydrophilic acrylic lenses inserted into the capsular bag at the end of the procedure.

The differences in the mydriasis between the two groups were compared using the unpaired $t$-test and Levene's test for equality of variance. A $P$-value of $<0.05$ was considered significant.

\section{Results}

A total of 150 patients in group A were dilated with the cocktail regimen, and 75 patients in group B were dilated using the conventional topical drops regimen. The male/ female ratio was comparable in both groups with group A having 72:78 and group B having 39:36. The mean age in group A was 58 years and in group B was 55 years. In group A, 22 patients (15\%) had a predisposing factor contributing to poor mydriasis, such as diabetes or pseudoexfoliation, and in group B, 12 patients (16\%) had similar predisposing factors.

A total of 18 patients (12\%) in group A and 15 patients $(20 \%)$ in group B were classified into the poor mydriasis. In all, 108 patients (72\%) in group A and 52 patients $(69 \%)$ in group B were in the moderate mydriasis category. A total of 24 patients $(16 \%)$ in group A and 8 patients $(10 \%)$ in group B were in the good mydriasis category. Thus, both groups were comparable (Table 1).

The dilation in both groups was evaluated at 15, 30, and $45 \mathrm{~min}$. At the $15 \mathrm{~min}$ mark, there was no statistical difference between the two groups. At $30 \mathrm{~min}$, a significant difference of $1.57 \mathrm{~mm}$ was seen between the two groups $(P<0.001)$. The average dilation in group $\mathrm{A}$ at $45 \mathrm{~min}$ was $7.13 \mathrm{~mm}$ and that in group B was $5.88 \mathrm{~mm}$. The difference achieved in both the dilating regimens $(1.25 \mathrm{~mm})$ was statistically significant $(P<0.001)$. The

Table 1 Levels of mydriasis in the two groups

\begin{tabular}{lrrr}
\hline Category of mydriasis & $\begin{array}{r}\text { Group A } \\
\text { (cocktail) }\end{array}$ & $\begin{array}{r}\text { Group B } \\
\text { (control) }\end{array}$ & P-value \\
\hline Poor mydriasis (5 mm) & $18(12 \%)$ & $15(20 \%)$ & 0.128 \\
Moderate mydriasis $(5.1-8 \mathrm{~mm})$ & $108(72 \%)$ & $52(69 \%)$ & 0.406 \\
Good mydriasis (>8 mm) & $24(16 \%)$ & $8(10 \%)$ & 0.063 \\
\hline
\end{tabular}

average intraoperative pupillary diameter noted after nucleus extraction in group A was $6.20 \mathrm{~mm}$ and in group $B$ was $5.28 \mathrm{~mm}$. This difference was also statistically significant $(P<0.001)$. The mean pupillary diameter noted after IOL implantation was $5.79 \mathrm{~mm}$ in group A and $4.38 \mathrm{~mm}$ in group B. This difference of $1.41 \mathrm{~mm}$ was also statistically significant using the unpaired $t$-test for equality of means.

The average pupillary diameter at the end of $45 \mathrm{~min}$, in the subset of patients with diabetes and PXF, was $7.47 \mathrm{~mm}$ in the cocktail group and $5.55 \mathrm{~mm}$ in the control group. This difference of $1.92 \mathrm{~mm}$ did not attain statistical significance due to the small sample size of the subset. Pupillary diameter at the time of nucleus delivery as well as IOL implantation was better in the cocktail group, but did not attain statistical significance either due to the small sample size.

Pulse, blood pressure, and pulse oximetry were measured pre- and postoperatively. There were no adverse cardiovascular events. The single operating surgeon was interviewed postoperatively regarding any loss of corneal clarity, and there were no such occurrences. Figures 2a (cocktail) and 2b (dilating drops) show gradual progression of mydriasis in the two groups.
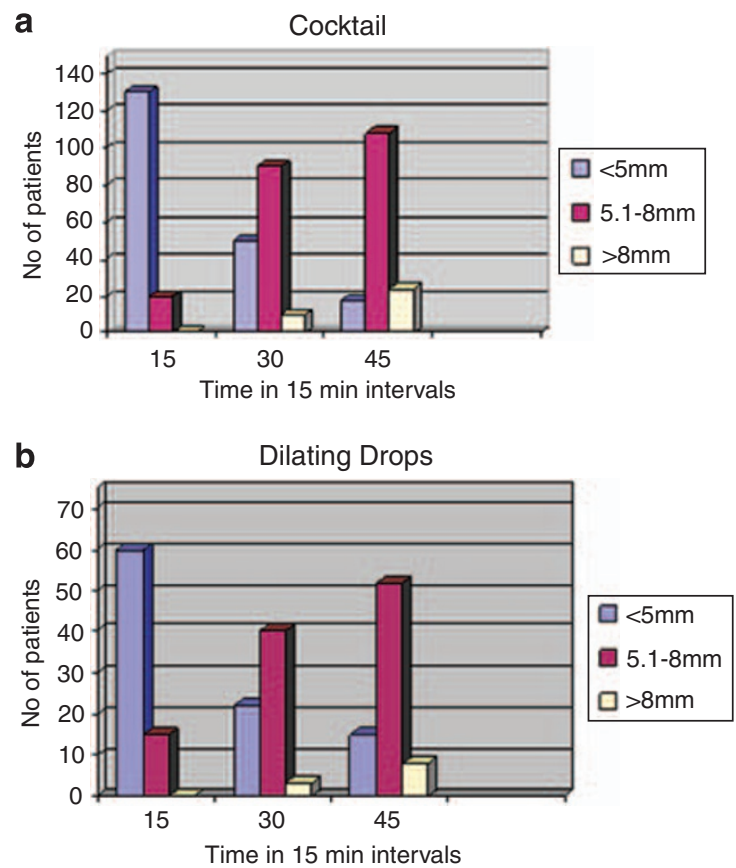

Figure 2 The gradual progression of mydriasis in the cocktail and control groups are shown. (a) cocktail; (b) dilating drops. 


\section{Discussion}

The presence of good mydriasis is a prerequisite for cataract surgery. Many methods have been suggested to overcome the problem of poor mydriasis. Adequate use of preoperative mydriatics, subconjunctival mydricaine, preservative free intracameral adrenaline 1, iris retractors, and sphincterotomies are some of the methods to combat poor mydriasis. Although all the above methods have been used at some point or another in cataract surgery world wide, the problem of poor mydriasis seems to be the bugbear of most surgeons. Hence, we undertook this study creating a new 'mydriatic cocktail' regimen to enable us to achieve better mydriasis before cataract surgery. This problem of poor mydriasis is commonly encountered in diabetics and patients with PXF syndrome. Because of a small pupil, the capsulorrhexis is inadvertently smaller giving rise to the contracted capsule syndrome, which is particularly common in diabetics. ${ }^{16}$ This can not only causes IOL decentration but also hamper the visualisation of the retina, which is a very important examination in diabetics. Most cases of PXF were present in the sixth and seventh decades, and most patients have nuclear sclerosis as well. Presence of unstable zonules, compounded with the factor of poor mydriasis, may further complicate cataract surgery in PXF. The problem of capsular contraction is common as well. ${ }^{17,18}$

All the above drugs used in our regimen have been proved to act singly as well as in combination to achieve good pupillary dilation. ${ }^{3,4,10}$ It was documented that the pupils dilated with cyclopentolate 1 day before surgery showed a mean reduction in subsequent mydriasis by $0.73 \mathrm{~mm}$ on the day of surgery compared with pupils that had been dilated with tropicamide. ${ }^{9}$ However, our study shows no pupillary fatigue with cyclopentolate. Pupil dilation with $2.5 \%$ phenylephrine alone, if allowed at least $40 \mathrm{~min}$ to act, is as satisfactory for the identification of cataracts in a normal population as $10 \%$

phenylephrine and 1\% tropicamide. Moreover, it is more acceptable because of reduced problems with glare and accommodation. ${ }^{13} \mathrm{~A}$ total of $2.5 \%$ phenylephrine has also been used to dilate eyes before cataract surgery and has been found to be efficacious. ${ }^{6}$ The systemic absorption and side effects are lower than thosewith the $10 \%$ formulation, yet retaining good mydriatic properties. We used $2.5 \%$ phenylephrine to reduce systemic side effects. Topical flurbiprofen has proven value in the maintenance of intraoperative mydriasis. ${ }^{8}$ Moxifloxacin of $0.5 \%$ was considered by us as the antibiotic of choice because of its preservative free, neutral $\mathrm{pH}$ status, and because of its excellent broad spectrum coverage. Hence, all the above drugs were considered in the cocktail.
A similar study to ours was carried out by Ong-Tone ${ }^{11}$ using a $4 \mathrm{~mm}$ wick for $10 \mathrm{~min}$. It showed that the wick delivers better quality of mydriasis compared with drops. However, it was not randomized, and no formal pupillary measurements were performed.

Another trial carried out by McCormick et $a l^{12}$ reported that the pupil dilatation before cataract surgery can be safely and effectively achieved using a triangular $3 \mathrm{~mm}$ pledget sponge soaked in mydriatic drugs. This trial did not find any statistically significant difference in the mydriasis achieved with the pledget and the traditional regimen of drops contrary to our study. One possible explanation is the duration for which the wick was used. We found maximal mydriasis to be achieved around the $30 \mathrm{~min}$ range, and have evaluated pupillary diameter at $45 \mathrm{~min}$ as opposed to $20 \mathrm{~min}$ used by the previous study.

The main complexity with instilling drops is that the average volume per drop is variable with the kind of dropper used. Further, the angle of dispensing the drop has a bearing on each drop's volume. An acute angle of 45 degree or less could mean a smaller volume drop with consequently lesser delivery. ${ }^{14,15}$ The use of the standard size wick could perhaps solve some of the problems associated with drop volume.

This study shows that the dilatation achieved was superior with the cocktail regimen, and the results obtained were statistically significant. Further, dilation with the cocktail reduced the cost to a third of that incurred using drops. The patient comfort and compliance with the cocktail was better owing to the single application and its less cumbersome nature. The main potential benefit of using this regimen is the reduction in nursing time required to administer drops. The nurses were initially apprehensive about using the wick. But they mastered the technique quickly and have appreciated the time it saves and the efficiency with which it works. The difference between the two groups was statistically as well as clinically apparent at the 30 min mark, but maximal mydriasis was attained only at 45 min. Drawbacks of this study are the lack of formal documentation of the levels of patient comfort using a formal questionnaire and the potential risk of contamination of the cocktail inherent to the study design. Significant results were not obtained in the diabetic and PXF subgroups due to small sample size. Further studies are needed in larger groups of patients with diabetes and PXF for more definitive results. Our experience suggests that the cocktail regimen is much better in maintaining intraoperative mydriasis in patients with diabetes and PXF.

In conclusion, the cocktail regimen is safe, efficacious, time saving, and cost effective. It is the dream of every surgeon to have eyes with excellent mydriasis to facilitate 
a complication-free cataract surgery. We hope that the above-mentioned mydriatic cocktail regimen may go a long way in this regard.

\section{References}

1 Behndig A, Eriksson A. Evaluation of surgical performance with intracameral mydriatics in phacoemulsification surgery. Acta Ophthalmol Scand 2004; 82: 144-147.

2 Lee YC, Millard J, Negvesky GJ, Butrus SI, Yalkowsky SH. Formulation and in vivo evaluation of ocular insert containing phenylephrine and tropicamide. Int J Pharm 1999; 182: 121-126.

3 Hirowatari T, Tokuda K, Kamei Y, Miyazaki Y, Matsubara M. Evaluation of a new pre operative ophthalmic solution. Can J Ophthalmol 2005; 40: 58-62.

4 Hirowatari T, Tokuda K, Kamei Y, Miyazaki Y, Matsubara M. TPD ophthalmic solution (mixture of tropicamide, phenylephrine hydrochloride and diclofenac sodium) for cataract surgery. Nippon Ganka Gakkai Zasshi 2002; 106: 630-633.

5 Sachdev MS, Singh K, Talwar D, Gupta SK, Dada VK. Comparative efficacy of diclofenac and flurbiprofen for maintenance of pupillary dilation during cataract surgery. Oph Surg 1994; 25: 92-94.

6 Ozturk F, Kurt E, Inan UU, Ilker SS. The efficacy of 2.5\% phenylephrine and flurbiprofen combined in inducing and maintaining pupillary dilation during cataract surgery. Eur J Ophthalmol 2000; 10: 144-148.

7 Hessener V, Schmidt K, Jacobi A. Anti inflammatory effects and aqueous humour concentration of various non steroidal anti inflammatory drugs in extra capsular surgery. Klin Mongrsbl Augenhielkd 1996; 208: 161-166.

8 Thaller VT, Kulshrestha MK, Bell K. The effect of pre-operative topical flurbiprofen or diclofenac on pupillary dilation. Eye 2000; 14: 642-645.

9 Power WJ, Hope-Ross M, Mooney DJ. Preoperative pupil fatigue. JCRS 1992; 18: 306-309.

10 Eyeson-Annan ML, Him LW, Battistuta D, Green A. Comparison of pupillary dilation using phenylephrine alone or in combination with tropicamide. Ophthalmology 1998; 105: 726-732.

11 Ong-Tone L. Use of wick to deliver pre operative mydriasis for cataract surgery. JCRS 2003; 29: 2060-2062.

12 McCormick A, Srinivasan S, Harun S, Watts M. Pupil dilation using a pledget sponge: a randomized control trial. Clinical and Expt ophthalmol 2006; 34: 545-549.

13 Yospaiboon Y, Luanratanakorn P, Noppawinyoowong C. Randomized Double-blind Study of Phenylephrine 2.5\% vs 10\% on Pupillary Dilation. J Med Assoc Thai 2004; 87: 1380-1384.

14 German EJ, Hurst MA, Wood D. Reliability of drop size from multi-dose eye drop bottles: is it cause for concern. Eye 1999; 13: 93-100.

15 Sklubalova Z, Zatloukal Z. Systematic study of factors affecting eye drop size and dosing variability. Pharmazie 2005; 60: 917-921.

16 Kato S, Oshika T, Numaga J, Hayashi Y, Oshiro M, Yuguchi T et al. Anterior capsular contraction after cataract surgery in eyes of diabetic patients. Br J Ophthalmol 2001; 85: 21-23.

17 Rakowska E, Zarnowski T, Zagorski Z. Capsular contraction syndrome. Klin Oczna 1999; 101: 375-378.

18 Davison JA. Capsule contraction syndrome. JCRS 1993; 19 : 582-589. 